\title{
Converging measures of workload capacity
}

\author{
Ami Eidels, Chris Donkin, Scott D. Brown, And Andrew Heathcote \\ University of Newcastle, Newcastle, New South Wales, Australia
}

\begin{abstract}
Does processing more than one stimulus concurrently impede or facilitate performance relative to processing just one stimulus? This fundamental question about workload capacity was surprisingly difficult to address empirically until Townsend and Nozawa (1995) developed a set of nonparametric analyses called systems factorial technology. We develop an alternative parametric approach based on the linear ballistic accumulator decision model (Brown \& Heathcote, 2008), which uses the model's parameter estimates to measure processing capacity. We show that these two methods have complementary strengths, and that, in a data set where participants varied greatly in capacity, the two approaches provide converging evidence.
\end{abstract}

Choices are often made on the basis of multiple attributes of an object or objects. An important question in this context is what happens to people's capacity for information processing as workload - the number of to-beprocessed stimuli-increases. Workload capacity is commonly measured using the redundant-target detection task (e.g., Miller, 1982, 1991; Townsend \& Nozawa, 1995). In this task, participants are presented with two targets (denoted as the redundant-target or "AB" condition), one target (either A or B), or no targets. Typically, the order of display conditions (i.e., no, one, or two targets) is randomized within blocks of trials, and participants are asked to respond positively if they detect either target and negatively if they observe neither target. Workload capacity can be assessed by comparing the time to make positive responses in the redundant- and single-target conditions.

Standard analyses, such as mean response time (RT) comparisons, provide ambiguous evidence about whether adding additional targets (i.e., increasing workload) reduces, improves, or has no effect on the efficiency of processing. For example, in a system where each stimulus is processed in parallel by separate and independent channels, mean RT reduces as the number of target stimuli increases, apparently indicating an increase in capacity, when capacity is in fact unlimited in the sense that processing in each channel is unaffected by processing in other channels. ${ }^{1}$ To avoid such ambiguities, Townsend and Nozawa (1995) developed systems factorial technology, a set of nonparametric analyses based on the entire distribution of RTs for correct responses (for applications, see Eidels, Townsend, \& Algom, 2010; Eidels, Townsend, \& Pomerantz, 2008; Wenger \& Townsend, 2001).

Here we elaborate a parametric model of rapid choice, the linear ballistic accumulator (LBA; Brown \& Heath- cote, 2008), to provide an alternative perspective on workload capacity. Brown and Heathcote (2008) showed that the LBA provides a comprehensive account of benchmark phenomena in single-target rapid-choice tasks. Moreover, the LBA is a psychologically plausible process model, with interpretable parameters that account for the quality of sensory input and for participants' response strategy. Because of its mathematical simplicity, the LBA has already been applied in areas ranging from the neural basis of rapid choice (e.g., Ho, Brown, \& Serences, 2009) to eye movements (Ludwig, Farrell, Ellis, \& Gilchrist, 2009). We take advantage of that simplicity to extend the LBA to the redundant-target task. We show that nonparametric and parametric approaches have complementary advantages that together provide converging evidence about workload capacity in cognitive systems.

\section{The Capacity Coefficient: A Nonparametric Measure}

Townsend and Nozawa's (1995) capacity coefficient, $C(t)$, compares a measure of the entire distribution of RT for correct responses in the double-target condition and the single-target conditions. The measure is based on the survivor function, $S(t)=1-F(t)$, where $F(t)$ is the cumulative density function, the probability of a response occurring before time $t$. For an OR redundant-target task, where one or the other target is sufficient for a positive response,

$$
C(t)=\frac{\log \left[S_{\mathrm{AB}}(t)\right]}{\log \left[S_{\mathrm{A}}(t) \cdot S_{\mathrm{B}}(t)\right]} .
$$

If $C(t)=1$ for all values of $t$, a system of parallel channels has unlimited capacity, such that processing in a given 
channel is unaffected by the increase in the number of targets. If $C(t)<1$, capacity is limited, such that increasing the number of targets takes a toll on each channel's performance. If $C(t)>1$, the system has super capacity: The processing efficiency of individual channels increases with increased workload. Typically, a plot of $C(t)$ is made for a middle range of $t$ (since the measure is unstable at the extremes), and overall capacity is judged subjectively, perhaps with the aid of a confidence region.

The capacity coefficient is very generally applicable, because its derivation does not rely on an assumed parametric form for correct RT distribution. However, in practice, the capacity coefficient may be confounded by differences in "base time" (i.e., the time to complete early sensory and response preparation and execution processes) and speed-accuracy trade-off between conditions contributing to $C(t)$. Townsend and Honey (2007) investigated base time effects and concluded that they are unlikely to be problematic. ${ }^{2}$ Similarly, the design used in the OR redundant-target task, where double- and single-target conditions are randomized within blocks of trials, makes confounding from speed-accuracy trade-off unlikely, since it is usually assumed that participants can change trade-off strategies only if they can anticipate upcoming conditions (Ratcliff, 1978).

In the next section, we introduce the standard LBA model and show how it can be extended to the redundanttarget task in terms of parameters quantifying the efficiency or rate of information processing, base time, and response caution, which controls speed-accuracy tradeoff. The latter two parameters allow us to account for effects that might confound the nonparametric capacity measure. Although such confounding is unlikely in the particular paradigm we examine here, the protection offered by modeling these effects could be useful in other paradigms. We then propose a measure of workload capacity based on rate parameters from double- and singletarget conditions. A second advantage of our parametric approach is that it provides an objective method of identifying whether capacity is limited, unlimited, or super.

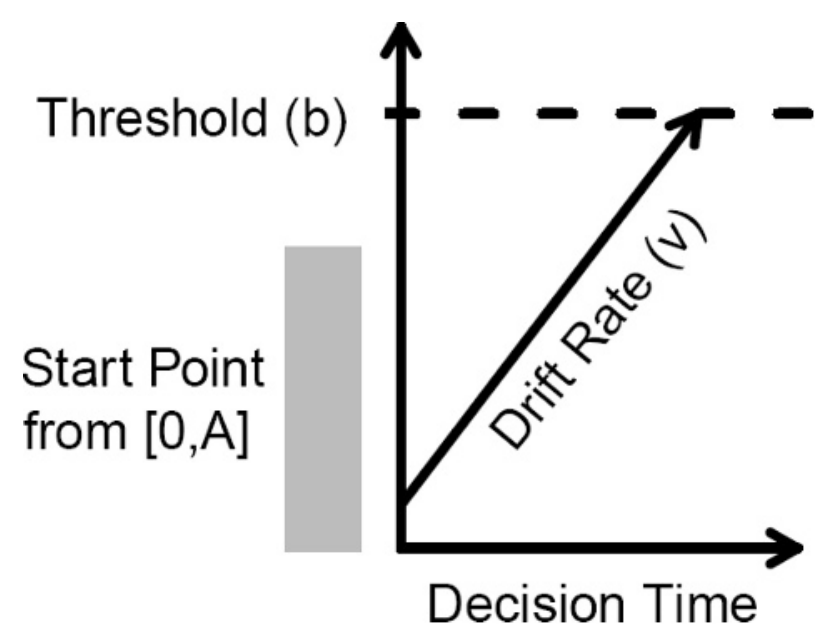

Figure 1. Illustration of a single linear ballistic accumulator.

\section{A Parametric Measure of Capacity}

As with most models of rapid choice, the LBA assumes that evidence about a decision is accumulated until it exceeds a threshold that controls response caution. Figure 1 illustrates a single LBA evidence accumulator. On each trial, the initial level of evidence in an accumulator is drawn from a uniform distribution with a zero minimum and a maximum determined by the parameter $A .{ }^{3}$ The accumulator's drift rate is drawn from a normal distribution with mean $v$ and standard deviation $s$. Evidence in the accumulator increases linearly, at a speed given by the drift rate, until it reaches a response threshold determined by parameter $b$. RT is the time taken for the evidence to reach threshold plus base time, which we model as a constant, $t_{0}$. Hence, the behavior of each accumulator is controlled by five parameters, $\boldsymbol{\theta}=\left(b, A, v, s, t_{0}\right)$. Closed-form solutions for the cumulative density, $F(t \mid \boldsymbol{\theta})$, and density, $f(t \mid \boldsymbol{\theta})$, of the time taken for a single accumulator to reach threshold are given in Brown and Heathcote (2008).

To model a choice between $N$ alternatives, the LBA uses $N$ independent accumulators. The predicted choice (and RT) is given by whichever accumulator reaches threshold first. The density functions ( $f$ and $F$ ) can be combined to obtain the joint density over response choices and times: The likelihood that response $i$ occurs at time $t$ is given by the product of the probability that no other accumulator has reached its threshold before $t$,

$$
\prod_{j \neq i}\left[1-F\left(t \mid \boldsymbol{\theta}_{j}\right)\right],
$$

and the density at time $t$ for accumulator $i$.

\section{LBA Redundant-Target Task Model}

The same approach can be used to apply the LBA to the redundant-target task, but there is a complication: Decisions about the two targets (A and B) require four accumulators, but there are only two responses ("neither target present" or "either target present"). The four accumulators collect evidence about whether (1) Target A is present, (2) Target A is absent, (3) Target B is present, and (4) Target $B$ is absent. We denote these accumulators $\mathrm{A}, \sim \mathrm{A}, \mathrm{B}$, and $\sim \mathrm{B}$. To resolve the complication, we propose a model in which a negative response occurs if both accumulators $\sim \mathrm{A}$ and $\sim \mathrm{B}$ reach threshold while both $\mathrm{A}$ and $\mathrm{B}$ are below threshold. ${ }^{4}$ The probability that both $\mathrm{A}$ and $\mathrm{B}$ are below threshold is given by the product of their survivor functions. The likelihood that $\sim \mathrm{A}$ terminates at $t$ after $\sim \mathrm{B}$ has already terminated is given by the product of their corresponding density and cumulative density, respectively, and similarly for the likelihood that $\sim \mathrm{B}$ terminates at $t$ after $\sim$ A has already terminated. Summing the likelihoods for these two mutually exclusive scenarios produces the overall likelihood of a negative response:

$$
\begin{aligned}
L(\mathrm{NO}, t)=S_{\mathrm{A}}(t) & \cdot S_{\mathrm{B}}(t) \\
\cdot & {\left[f_{\sim \mathrm{A}}(t) \cdot F_{\sim \mathrm{B}}(t)+f_{\sim \mathrm{B}}(t) \cdot F_{\sim \mathrm{A}}(t)\right] . }
\end{aligned}
$$

A positive, "either target present" response occurs if either A or B reaches threshold while $\sim \mathrm{A}, \sim \mathrm{B}$, or both remain below threshold. Hence, the likelihood of a positive response at time $t$ is again the sum of the likelihoods of 
two scenarios (i.e., A reaches threshold and B has not, and vice versa):

$$
\begin{aligned}
L(\mathrm{YES}, t)= & {\left[1-F_{\sim \mathrm{A}}(t) \cdot F_{\sim \mathrm{B}}(t)\right] } \\
& \cdot\left[f_{\mathrm{A}}(t) \cdot S_{\mathrm{B}}(t)+f_{\mathrm{B}}(t) \cdot S_{\mathrm{A}}(t)\right] .
\end{aligned}
$$

Equations 2 and 3 are used to evaluate the likelihood of all responses for a given set of parameters for each condition, enabling the most likely parameter set to be found by an optimization algorithm. Note that these estimates are constrained by all aspects of the data, including accuracy and error RTs, not just correct RTs as is the case for the nonparametric capacity measure.

We propose that workload capacity can be measured by comparing drift rates for single- and double-target conditions. This approach assumes that potential interactions between stimulus-processing channels, which determine capacity, occur before the decision stage and influence the drift-rate input to the LBA. Capacity is called "unlimited" if each accumulator operates at the same rate regardless of whether a signal is presented for processing by other accumulators. If the accumulation rate decreases or increases as more signals are added, capacity is considered limited or super, respectively. We test this new definition for workload capacity, based on the relative magnitude of single- versus double-target drift rates, by comparing it with $C(t)$ measures for individual participants in Eidels et al.'s (2008) Experiment 3.

\section{Application}

In their third experiment, Eidels et al. (2008) presented their participants with line segments that, when presented together, created an open or closed form (e.g., "L" and ")" together form a closed triangle). Of the four line segments used, two were designated as targets. For example, “/” and a backward "L" might be labeled A and B, whereas "l" and "L" might be labeled $\sim \mathrm{A}$ and $\sim \mathrm{B}$, respectively. Partici-

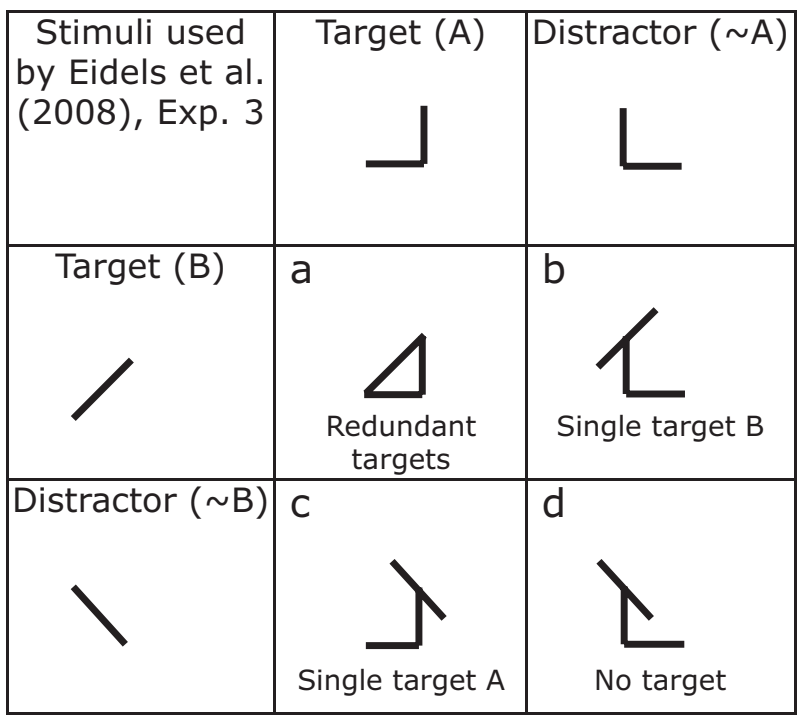

Figure 2. The stimuli used by Eidels, Townsend, and Pomerantz (2008) in their Experiment 3.
Table 1

Summary of the Mapping Between Possible Linear Ballistic Accumulator Drift-Rate Parameters in a RedundantTarget Task (Left-Hand Side) and the Simplified Set of Five

\begin{tabular}{|c|c|c|c|}
\hline & \multicolumn{2}{|c|}{ Target A } \\
\hline & & Presented (A) & Not Presented $(\sim \mathrm{A})$ \\
\hline \multirow{8}{*}{ Target B } & \multirow{4}{*}{ Presented (B) } & $v_{\mathrm{A} \mid \mathrm{AB}}=v_{\mathrm{DT}}$ & $v_{\mathrm{A} \mid \sim \mathrm{AB}}=v_{\sim \mathrm{T}}$ \\
\hline & & $v_{\sim \mathrm{A} \mid \mathrm{AB}}=v_{\mathrm{NT}}$ & $v_{\sim \mathrm{A} \mid \sim \mathrm{AB}}=v_{\sim \mathrm{NT}}$ \\
\hline & & $v_{\mathrm{B} \mid \mathrm{AB}}=v_{\mathrm{DT}}$ & $v_{\mathrm{B} \mid \sim \mathrm{AB}}=v_{\mathrm{ST}}$ \\
\hline & & $v_{\sim \mathrm{B} \mid \mathrm{AB}}=v_{\mathrm{NT}}$ & $v_{\sim \mathrm{B} \mid \sim \mathrm{AB}}=v_{\mathrm{NT}}$ \\
\hline & \multirow{4}{*}{ Not Presented $(\sim \mathrm{B})$} & $v_{\mathrm{A} \mid \mathrm{A} \sim \mathrm{B}}=v_{\mathrm{ST}}$ & $v_{\mathrm{A} \mid \sim \mathrm{A} \sim \mathrm{B}}=v_{\sim \mathrm{T}}$ \\
\hline & & $v_{\sim \mathrm{A} \mid \mathrm{A} \sim \mathrm{B}}=v_{\mathrm{NT}}$ & $v_{\sim \mathrm{A} \mid \sim \mathrm{A} \sim \mathrm{B}}=v_{\sim \mathrm{NT}}$ \\
\hline & & $v_{\mathrm{B} \mid \mathrm{A} \sim \mathrm{B}}=v_{\sim \mathrm{T}}$ & $v_{\mathrm{B} \mid \sim \mathrm{A} \sim \mathrm{B}}=v_{\sim \mathrm{T}}$ \\
\hline & & $v_{\sim \mathrm{B} \mid \mathrm{A} \sim \mathrm{B}}=v_{\sim \mathrm{NT}}$ & $v_{\sim \mathrm{B} \mid \sim \mathrm{A} \sim \mathrm{B}}=v_{\sim \mathrm{NT}}$ \\
\hline
\end{tabular}
Parameters Used in Estimation (Right-Hand Side)

Note-For the redundant-target task, subscripts indicate the corresponding accumulator and experimental condition. For example, $v_{\mathrm{A} \mid \mathrm{A} \sim \mathrm{B}}$ is the drift rate for the accumulator that detects the presence of Target A when Target A was presented but Target B was not. Subscripts for the simplified set of five parameters are explained in the main body of the article.

pants were presented with four possible forms, illustrated in Figure 2, which occurred equally often over trials: One form contained both target line segments (redundant targets), two forms contained only one of the targets, and one form contained neither target.

To avoid bias in drift-rate estimates and, hence, in our parametric capacity measure, it is important to consider whether LBA parameters other than drift rate vary with the number of targets. For example, the stimulus encoding component of base time might decrease with two targets versus one target due to perceptual factors. To account for such possibilities, we estimated separate base time parameters for the double-, single-, and nontarget conditions$t_{0 \mathrm{DT}}, t_{0 \mathrm{ST}}$, and $t_{0 \mathrm{NT}}$, respectively. In contrast, following the convention used since Ratcliff (1978), we assumed equal response criteria for the single- and double-target conditions because their order was randomized within blocks of trials. Trials requiring a positive response were more common $(75 \%)$ than trials requiring a negative response, so participants may be biased toward making positive responses. To model this, we estimated one threshold parameter common to both target accumulators $\left(b_{\mathrm{T}}\right)$ but a different common parameter for the nontarget accumulators $\left(b_{\mathrm{NT}}\right)$.

Because there are four accumulators (one for the presence and one for the absence of Target A, and similarly for Target B) and four experimental conditions (redundant target, $\mathrm{AB}$; single target, $\mathrm{A} \sim \mathrm{B}$ or $\sim \mathrm{AB}$; and no target, $\sim \mathrm{A} \sim \mathrm{B}$ ), there could be up to 16 drift-rate $(v)$ parameters. As illustrated in Table 1, simplifying assumptions reduced the 16 possible drift rates to just five free parameters. These assumptions result in the simplest reasonable model that still allows for the estimation of capacity within a redundant-target task. First, we assumed that drift rates were equivalent for $\mathrm{A}$ and $\mathrm{B}$ stimuli and for $\sim \mathrm{A}$ and $\sim \mathrm{B}$ stimuli. This assumption need not be true, but when we repeated our analyses dropping this assumption where possible, we found similar results. ${ }^{5}$ Two free parameters describe the drift rates when a target is absent: one parameter for the nontarget accumulator, $v_{\sim \mathrm{NT}}$, and one for 
Table 2

Average Parameter Values for the Super-Capacity (Based on Data From 7 Participants), Unlimited-Capacity (8), and Limited-Capacity (9) Participants, Grouped As Detailed in the Text

\begin{tabular}{|c|c|c|c|c|c|c|c|c|c|c|c|}
\hline & $A$ & $t_{0 \mathrm{DT}}$ & $t_{0 \mathrm{ST}}$ & $t_{0 \mathrm{NT}}$ & $b_{\mathrm{T}}$ & $b_{\mathrm{NT}}$ & $v_{\mathrm{DT}}$ & $v_{\mathrm{ST}}$ & $v_{\mathrm{NT}}$ & $v_{\sim \mathrm{T}}$ & $v_{\sim \mathrm{NT}}$ \\
\hline Super & 1.00 & 0.15 & 0.14 & 0.12 & 1.64 & 1.76 & 2.96 & 2.52 & -0.60 & 0.56 & 2.68 \\
\hline Unlimited & 0.96 & 0.13 & 0.14 & 0.10 & 1.56 & 1.84 & \multicolumn{2}{|c|}{2.44} & -0.54 & 0.16 & 2.64 \\
\hline Limited & 0.76 & 0.14 & 0.16 & 0.10 & 1.28 & 1.68 & 2.28 & 2.72 & -0.04 & 0.24 & 3.36 \\
\hline
\end{tabular}

Note-Workload capacity is estimated via the difference between accumulation rates for double- and singletarget conditions. Positive, null, and negative values for $v_{\mathrm{DT}}-v_{\mathrm{ST}}$ imply super, unlimited, and limited capacity, respectively. For unlimited-capacity participants only, the model fit assumed $v_{\mathrm{DT}}=v_{\mathrm{ST}}$, so only a single value is reported in the table.

the target accumulator, $v_{\sim T}$. Subscripts T and NT denote whether the rate corresponds to a target or a nontarget accumulator, and the $\sim$ indicates that either $\sim \mathrm{A}$ or $\sim \mathrm{B}$ was presented. Three parameters set the drift rates when targets were present: one for the nontarget accumulators, $v_{\mathrm{NT}}$, and two for the target accumulators, depending on whether the display contained one (single-target condition, $v_{\mathrm{ST}}$ ) or both (double-target condition, $v_{\mathrm{DT}}$ ). We used the relative magnitudes of $v_{\mathrm{DT}}$ and $v_{\mathrm{ST}}$ as a measure of capacity. If the drift rate is larger in the double- than in the single-target condition, super capacity is indicated; if $v_{\mathrm{DT}}$ is smaller than $v_{\mathrm{ST}}$, limited capacity is indicated; and if the two are equal, unlimited capacity is indicated.

The final 2 parameters specify the trial-to-trial variability in starting points $(A)$ and drift rate $(s)$. We estimated a single value of $A$ across all responses and conditions. The $s$ parameter was treated as a scaling parameter and arbitrarily fixed at $s=1$ (see Donkin, Brown, \& Heathcote, 2009). Overall, 11 free parameters were used to fit RT distributions for correct and error responses for the four stimulus conditions. Maximum likelihood parameter estimates were found separately for each participant.

\section{Results}

Participants were grouped as having super (7 participants), unlimited (8), or limited (12) capacity, on the basis of drift-rate estimates. We first determined whether or not each participant demonstrated unlimited capacity by fitting two versions of the LBA model: one in which drift rate was equal in both single- and double-target conditions (i.e., $v_{\mathrm{DT}}=v_{\mathrm{ST}}$ ) and another in which the two parameters were allowed to differ. We then used the Bayesian information criterion (Schwarz, 1978) — a selection tool that determines the best model, taking into account both fit and model complexity - to determine which model was more appropriate, the one with one or the one with two drift-rate parameters. Any participant for whom one rate parameter was more appropriate was deemed to have unlimited capacity ( since the speed of evidence accumulation was equal in both single- and double-target conditions). Anyone requiring separate parameters for double- and single-target responses was classified as having super capacity (if $v_{\mathrm{DT}}>v_{\mathrm{ST}}$ ) or limited capacity (if $\left.v_{\mathrm{DT}}<v_{\mathrm{ST}}\right)$.

Table 2 contains average parameter estimates for each of the three groups of participants. As expected, the threshold estimates were greater for nontarget accumulators than for target accumulators, reflecting a response bias in favor of positive responses. Base time did not differ much between one- and two-target displays, but was systematically smaller for displays with no targets.

Figure 3 shows that this method for grouping participants produced plausible data patterns and model predictions. To create the figure, participants were first grouped according to their capacity classification, and data from all participants in each of the groups were combined such that three collective data sets were created, for limited, unlimited, and super capacity. Empirical RT distributions (histograms) were estimated using the combined data, and model predictions were generated, using average parameter values for each group. All participants, regardless of their observed capacity, made more errors and responded slower in the single-target condition than in the doubletarget condition. The difference between single- and double-target conditions, however, increased as participants exhibited higher capacity. In particular, participants classified as having limited capacity were slightly faster with double targets than with single targets (observed mean RTs were 0.50 and $0.56 \mathrm{sec}$, respectively), whereas participants classified as having super capacity were substantially faster with double targets than with single targets $(0.48$ vs. $0.66 \mathrm{sec})$. Response accuracy results followed a similar pattern. Participants with unlimited capacity sat between limited- and super-capacity participants in terms of the speed and accuracy of their responses, results consistent with what is known about the effects of workload capacity on performance.

The classification of participants on the basis of the relative magnitude of drift rates in the double- and single-target conditions also mostly agrees with results calculated using Townsend and Nozawa's (1995) capacity coefficient. Of the 27 participants, only 3 showed a discrepancy between the classifications provided by driftrate difference and $C(t)$. All 3 showed the same discrepancy: limited capacity based on drift difference and super capacity based on $C(t)$. This disagreement may arise from summarizing the continuous $C(t)$ function with a single number, given that the misclassified participants showed super capacity for relatively short ranges of $t$.

Figure 4 shows observed $C(t)$ plots for the remaining 24 participants, using the same groups as in Figure 3. Participants classified as limited capacity show $C(t)<1$ for a substantial portion of their $C(t)$ function. As expected, participants classified as unlimited capacity on the basis of drift rate tended to have $C(t)$ values close to unity. Similarly, participants classified as super capacity on the basis 
Limited

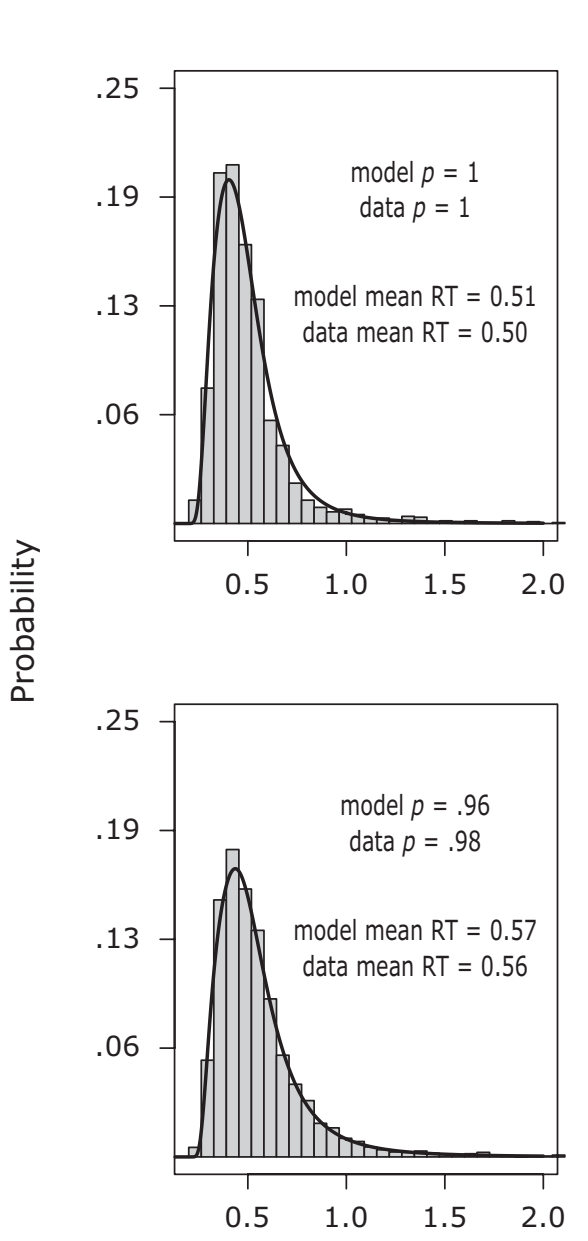

Unlimited

Double Target

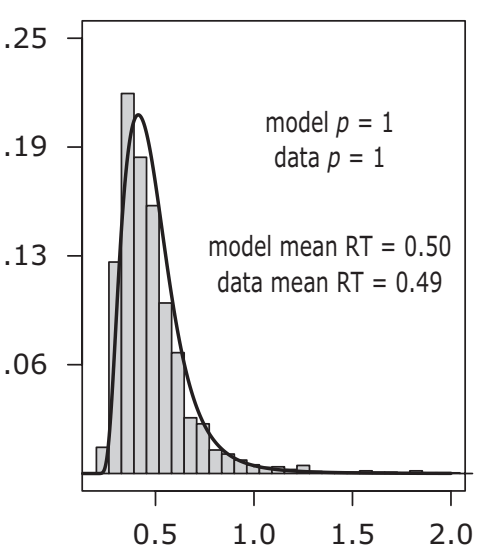

Single Target

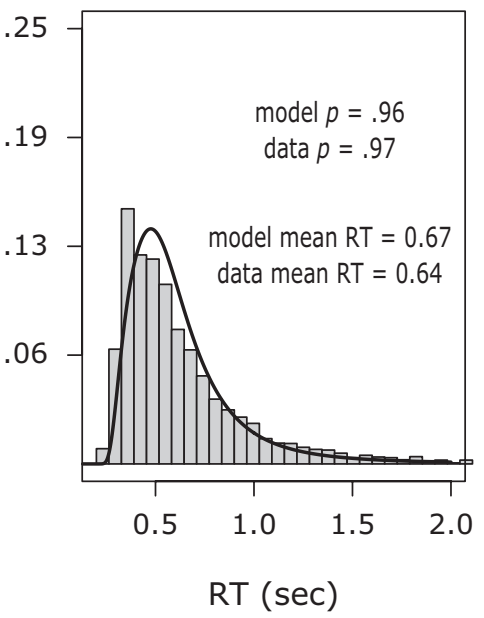

Super

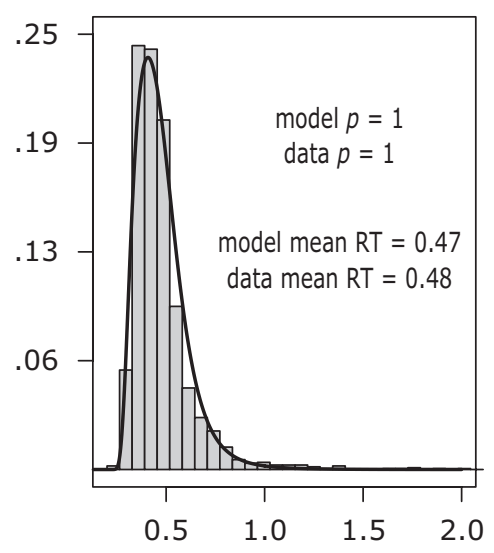

Figure 3. Results from fits of the linear ballistic accumulator to Experiment 3 data from Eidels et al. (2008). Histograms are empirically correct response time (RT) distributions, and thick black lines are model predictions. Plots are grouped into double- and singletarget conditions (top and bottom rows) and participants deemed to have limited, unlimited, and super capacity (left, center, and right columns) on the basis of accumulation rates. Each panel further indicates the proportion of correct responses and mean RT for the data and model.

of drift rates all show $C(t)>1$ for a substantial portion of their $C(t)$ function.

As a final demonstration of the agreement between the parametric and nonparametric methods, Figure 5 plots $C(t)$ estimates on the basis of predicted correct RT distributions from the LBA. To calculate the predicted $C(t)$ values, we took each individual's best-fitting parameters and simulated a set of 100,000 observations for each condition. We then calculated $C(t)$ for the simulated data, using the same methods as for the empirical data in Figure 4. Predicted and empirical $C(t)$ values in the two figures agree closely.

\section{Discussion}

We proposed a parametric method of assessing workload capacity that complements Townsend and Nozawa's (1995) nonparametric measure of capacity in the OR redundant-target task. In this task, participants respond positively if one or the other target stimulus is presented.
The nonparametric measure, $C(t)$, is calculated by comparing the distribution of the time taken to make correct responses to displays with one versus two targets. Our parametric approach is based on an evidence accumulation model of single-target choice tasks, the LBA (Brown $\&$ Heathcote, 2008), estimated on the basis of all responses in all conditions.

We first extended the LBA to model performance in the redundant-target task, then showed that the extended model provided an accurate account of the redundanttarget task data reported by Eidels et al. (2008) in terms of parameters that quantify base time, the drift rate (the rate of evidence accumulation), and the response threshold (the amount of evidence required for a response). Importantly, individual participants' capacity determined by the LBA model's drift-rate parameters in the single- and double-target conditions matched individual differences in estimates of $C(t)$. 


\section{Limited}

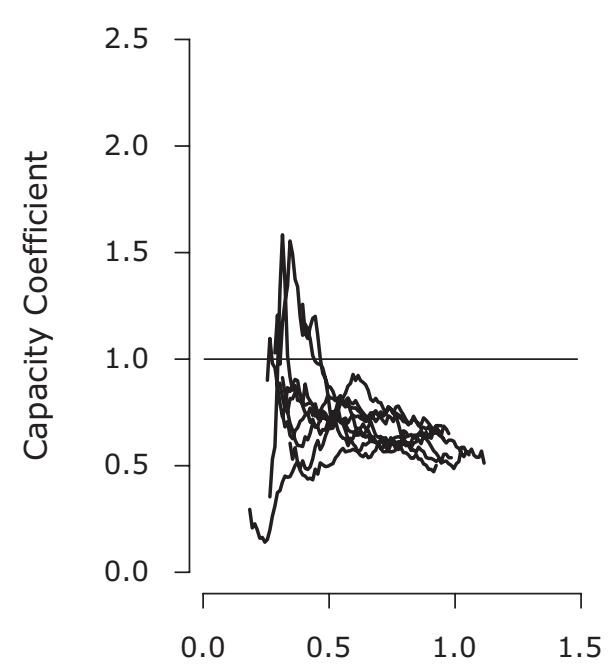

Unlimited

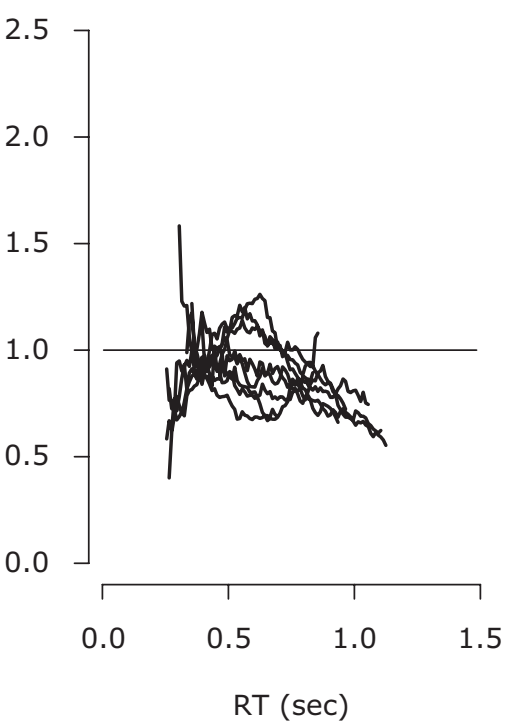

Super

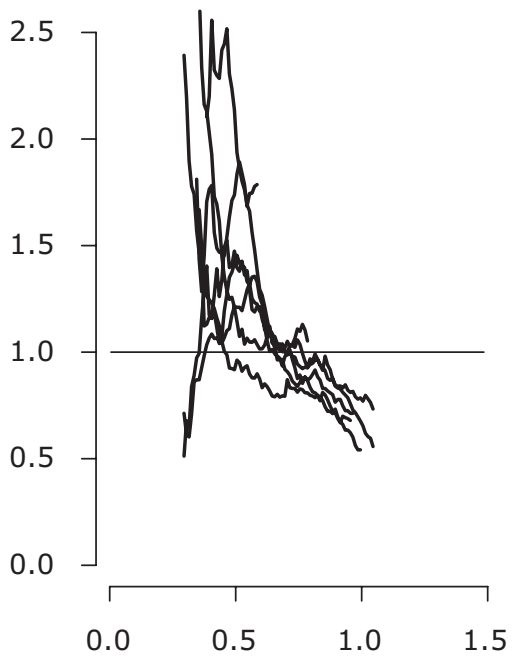

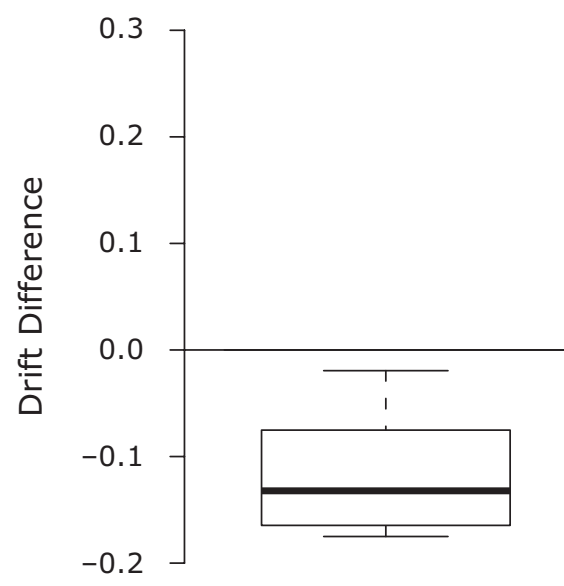
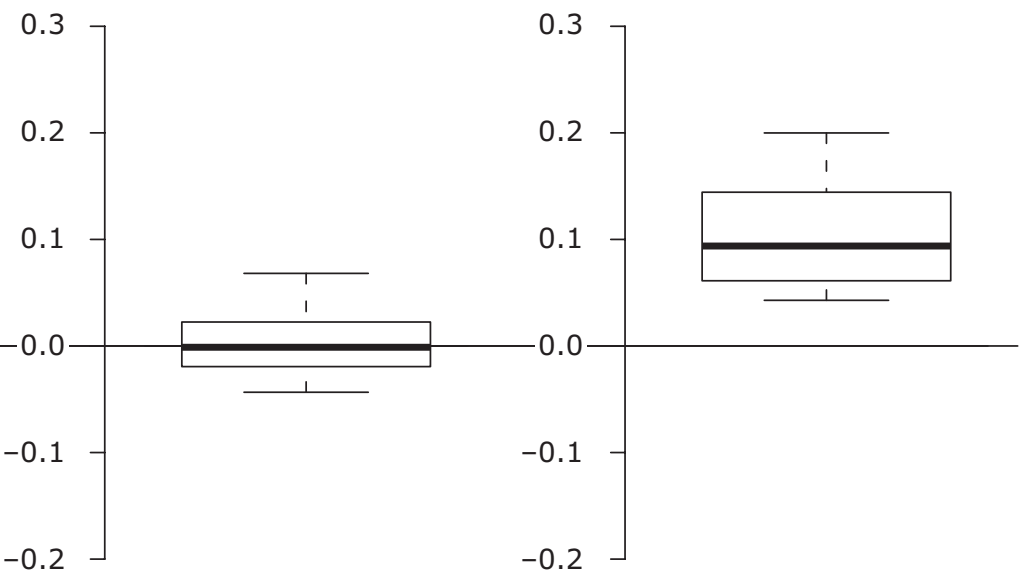

Figure 4. Capacity coefficients calculated from each individual's empirical response time (RT) distributions (top row of plots). Individuals have been grouped into limited, unlimited, and super capacity on the basis of the relative magnitudes of drift rates for doubleand single-target conditions. Boxplots of the distribution of drift-rate differences for each group are also shown in the second row of plots. The numbers of participants classified into each capacity group are 7, 8, and 12 for super, unlimited, and limited capacity, respectively.

As well as providing converging evidence about capacity, the parametric approach has the advantage of a formal method for classifying participants as having limited, unlimited, or super capacity. A further advantage applies in paradigms where the conditions that contribute to the nonparametric capacity measure differ in base time and/or response threshold. In the AND version of the redundanttarget paradigm, where a positive response is made only when both stimuli are targets, for example, different correct responses are required for single- and double-target conditions. Our results for the OR task (Table 2) indicated threshold and base time differences between accumulators responsible for collecting evidence for different types of responses. If this finding occurred in the AND task, the nonparametric $C(t)$ measure might be confounded. In contrast, our parametric approach would not be confounded, because these differences are modeled.
The LBA is one of several evidence accumulation models that provide an accurate account of benchmark phenomena in single-target rapid-choice paradigms. Similarly comprehensive models include Ratcliff's (1978) diffusion model and the stochastic (Usher \& McClelland, 2001) and ballistic (Brown \& Heathcote, 2005) leaky competing accumulator models. Any of these models could also be used to measure capacity analogously to the above methods. We used the LBA because it has closed-form analytic solutions that enable easy extension to the redundant-target task.

Assuming a particular evidence accumulation model makes our parametric approach less general than Townsend and Nozawa's (1995) nonparametric estimate. However, Donkin, Brown, Heathcote, and Wagenmakers (2010) found a direct mapping between the LBA and diffusion model drift-rate and base time estimates when the response threshold did not differ between conditions, as is likely the 
Limited

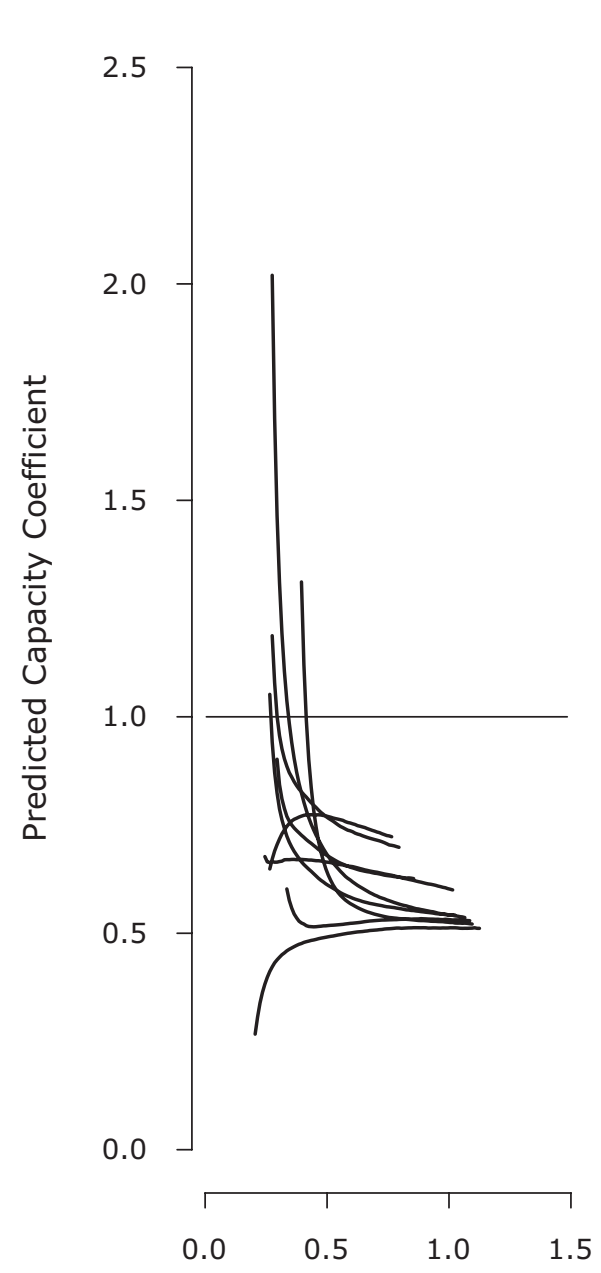

Unlimited

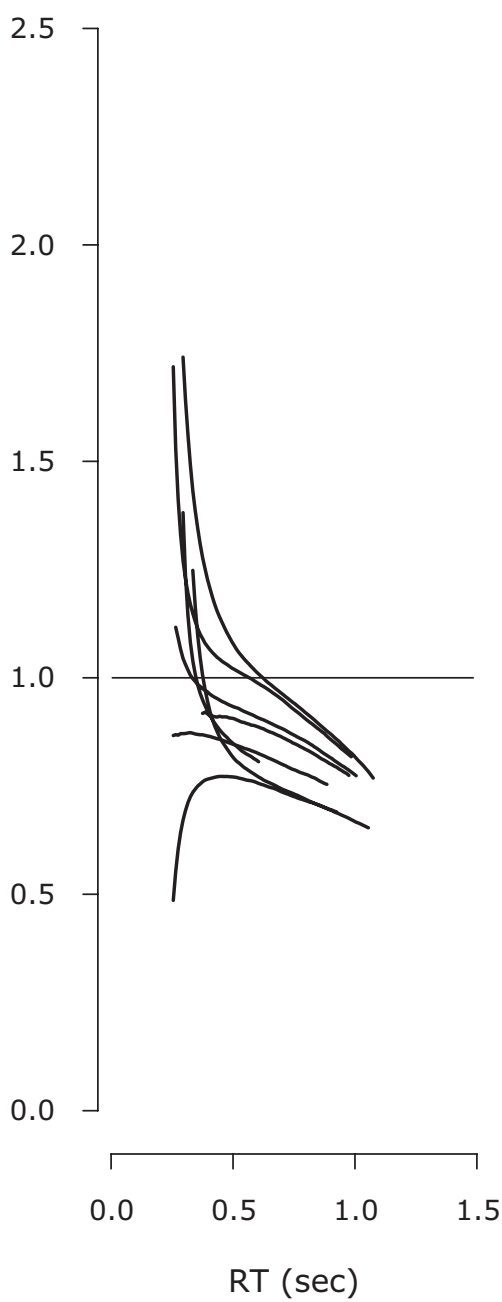

Super

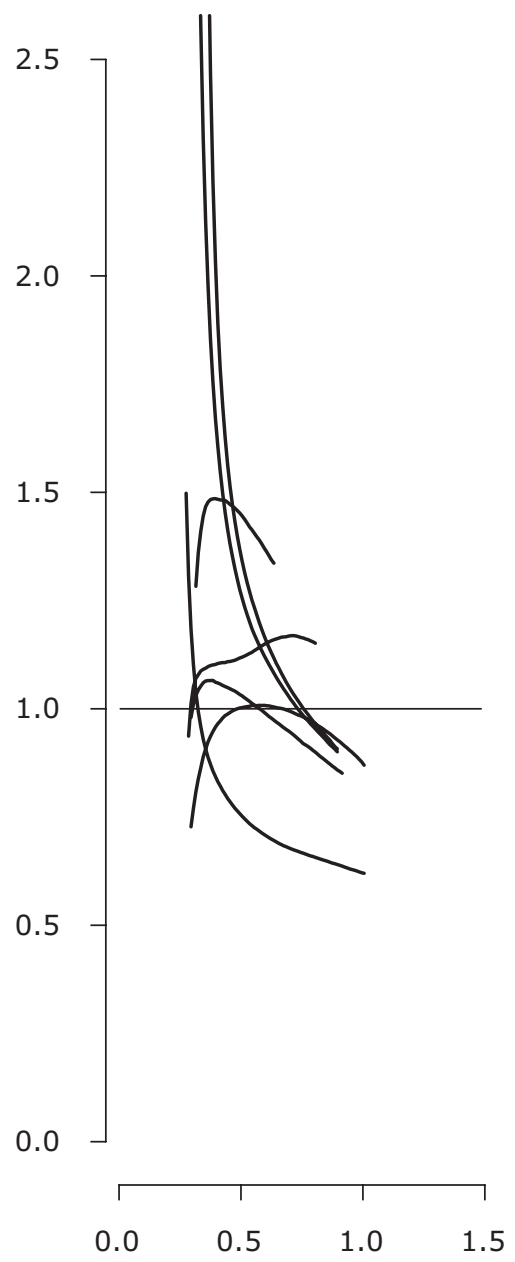

Figure 5. Capacity coefficients calculated from predicted response time (RT) distributions on the basis of each individual's bestfitting parameters of the linear ballistic accumulator.

case between single- and double-target conditions in the OR redundant-targets paradigm. Similarly, Van Ravenzwaaij and Oberauer (2009) found a strong correlation between diffusion and (stochastic) leaky competing accumulator parameter estimates. Hence, it seems likely that our LBA-based capacity estimate will be relatively general, at least within the class of evidence accumulator models.

\section{AUTHOR NOTE}

This study was supported by a Keats Endowment grant to A.E. We thank Joseph Houpt and two anonymous reviewers for insightful comments on earlier versions of this article. Correspondence should be addressed to A. Eidels, School of Psychology, University of Newcastle, Callaghan, NSW 2308, Australia (e-mail: ami.eidels@newcastle.edu.au).

Note-Accepted by Cathleen M. Moore's editorial team.

\section{REFERENCES}

Brown, S. [D.], \& Heathcote, A. (2005). A ballistic model of choice response time. Psychological Review, 112, 117-128.
Brown, S. D., \& Heathcote, A. (2008). The simplest complete model of choice response time: Linear ballistic accumulation. Cognitive Psychology, 57, 153-178.

Donkin, C., Brown, S. D., \& Heathcote, A. (2009). The overconstraint of response time models: Rethinking the scaling problem. Psychonomic Bulletin \& Review, 16, 1129-1135.

Donkin, C., Brown, S. D., Heathcote, A., \& Wagenmakers, E.-J. (2010). Diffusion versus linear ballistic accumulation: Different models for response time, same conclusions about psychological mechanisms? Manuscript submitted for publication.

Eidels, A., Townsend, J. T., \& Algom, D. (2010). Comparing perception of Stroop stimuli in focused versus divided attention: Evidence for dramatic processing differences. Cognition, 114, 129-150.

Eidels, A., Townsend, J. T., \& Pomerantz, J. R. (2008). Where similarity beats redundancy: The importance of context, higher order similarity, and response assignment. Journal of Experimental Psychology: Human Perception \& Performance, 34, 1441-1463.

Ho, T., Brown, S., \& Serences, J. (2009). Domain general mechanisms of perceptual decision making in human cortex. Journal of Neuroscience, 29, 8675-8687.

Ludwig, C. J. H., Farrell, S., Ellis, L. A., \& Gilchrist, I. D. (2009). The mechanism underlying inhibition of saccadic return. Cognitive Psychology, 59, 180-202. 
Miller, J. (1982). Divided attention: Evidence for coactivation with redundant signals. Cognitive Psychology, 14, 247-279.

MilleR, J. (1991). Channel interaction and the redundant-targets effect in bimodal attention in bimodal divided attention. Journal of Experimental Psychology: Human Perception \& Performance, 17, 160-169.

RAAB, D. (1962). Statistical facilitation of simple reaction time. Transactions of the New York Academy of Sciences, 43, 574-590.

Ratcliff, R. (1978). A theory of memory retrieval. Psychological Review, 85, 59-108.

SCHWARZ, G. (1978). Estimating the dimension of a model. Annals of Statistics, 6, 461-464.

Townsend, J. T., \& Honey, C. J. (2007). Consequences of base time for redundant signals experiments. Journal of Mathematical Psychology, 51, 242-265.

Townsend, J. T., \& Nozawa, G. (1995). Spatio-temporal properties of elementary perception: An investigation of parallel, serial and coactive theories. Journal of Mathematical Psychology, 39, 321-360.

TownsEnd, J. T., \& Wenger, M. J. (2004). A theory of interactive parallel processing: New capacity measures and predictions for a response time inequality series. Psychological Review, 111, 1003-1035.

Usher, M., \& MCClelland, J. L. (2001). The time course of perceptual choice: The leaky competing accumulator model. Psychological Review, 108, 550-592.

Van RavenzwaAis, D., \& Oberauer, K. (2009). How to use the diffusion model: Parameter recovery of three methods: EZ, fast-dm, and DMAT. Journal of Mathematical Psychology, 6, 463-473.

Wenger, M. J., \& Townsend, J. T. (2001). Faces as Gestalt stimuli: Process characteristics. In M. J. Wenger \& J. T. Townsend (Eds.), Com- putational, geometric, and process perspectives on facial cognition: Contexts and challenges (pp. 229-284). Mahwah, NJ: Erlbaum.

\section{NOTES}

1. When parallel channels race to determine the response, RTs on multiple-target trials can be faster than RTs on single targets via statistical facilitation (Raab, 1962). Miller (1982) proposed an upper bound on statistical facilitation for multiple channels involved in a race within the redundant target task - the race model inequality. He suggested that a violation of this inequality rules out all race models. Within our framework, the race model inequality is interpreted as a bound on capacity; its violation implies super capacity (Townsend \& Wenger, 2004).

2 . They showed that, assuming that base time is equal in distributions across single- and double-target conditions, $C(t)$ estimates become biased when base time accounts for a substantial proportion of total RT variance. Our parametric approach assumes constant base times that may differ between conditions. We assumed a nonvarying time, because typically (and in the application reported here) variable base times do not much improve the fit of the LBA. Freely estimating separate base times for different conditions allowed us to check, rather than assume, equality between conditions.

3. Parameter $A$ is italicized to distinguish it from a nonitalicized $\mathrm{A}$ used to denote an experimental stimulus.

4. The Appendix describes an alternative model. For the data analyzed here, both produced equivalent conclusions.

5. Since participants do not indicate which target they detected first in the double-target condition, in that condition separate drift rates for $\mathrm{A}$ and $\mathrm{B}$ stimuli are not identifiable.

\section{APPENDIX}

In this Appendix, we derive the likelihoods for YES and NO responses in the redundant target paradigm under an alternative model that treats the accumulator pairs looking at the same target as a single unit. In the model presented in the main text, all four accumulators $(\mathrm{A}, \mathrm{B}, \sim \mathrm{A}, \sim \mathrm{B})$ race to a single logical decision gate. Alternatively, one can model the same task as two subprocesses, each acting as a race between two accumulators. (1) Accumulators A and $\sim \mathrm{A}$ race to one OR gate, to determine whether Target A is presented. (2) Accumulators B and $\sim \mathrm{B}$ race to another OR gate, to determine whether Target $\mathrm{B}$ is presented. As a consequence, once the activation of one pair member crosses its threshold, the state of the other member becomes irrelevant. The outcomes of the two subprocesses then race to a final gate. Both models are useful, so we tested the alternative model and found that it completely agrees with the fits of the first model. The average difference between drift-rate differences (on double- vs. single-target conditions) in the first and second models was a negligible 1.7\%. Most importantly, the second model agrees with the first on the classification of individual participants to three capacity groups, for all 27 participants.

In the alternative model, there are four mutually exclusive ways that the system can make a YES response and two mutually exclusive ways that it can make a NO response, which together form an exhaustive set of potential outcomes.

\section{Likelihood of a YES Response}

Two of the ways that a YES response can be made require only the PDFs and CDFs on which Equations 2 and 3 are based:

1. At time $t, \mathrm{~A}$ is at threshold and all other accumulators are below threshold, with likelihood

$$
L_{1}(\mathrm{YES}, t)=f_{\mathrm{A}}(t) \cdot S_{\sim \mathrm{A}}(t) \cdot S_{\mathrm{B}}(t) \cdot S_{\sim \mathrm{B}}(t) .
$$

2. At time $t, \mathrm{~B}$ is at threshold and all other accumulators are below threshold, with likelihood

$$
L_{2}(\mathrm{YES}, t)=f_{\mathrm{B}}(t) \cdot S_{\sim \mathrm{B}}(t) \cdot S_{\mathrm{A}}(t) \cdot S_{\sim \mathrm{A}}(t) .
$$

The remaining four cases require a new expression for the probability of events occurring in pairs of accumulators treated as a single unit. The required expression is for the probability that one member of the pair (say, X) crossed its threshold at time $x<t$ and that the other pair member (say, Y) had not crossed its threshold at time $x$, $q(\mathrm{X}, \mathrm{Y}, t)$. For example, $\mathrm{X}$ might be the A accumulator and $\mathrm{Y}$ might be the $\sim \mathrm{A}$ accumulator, with corresponding probability $q(\mathrm{~A}, \sim \mathrm{A}, t)$. This probability is given by (numerically) integrating over $x \in(0, x)$ :

$$
q(\mathrm{X}, \mathrm{Y}, t)=\int_{0}^{t} f_{\mathrm{X}}(x) S_{\mathrm{Y}}(x) d x .
$$

With this term in hand, we can now write out the likelihoods for the two remaining YES cases: 


\section{APPENDIX (Continued)}

3. At time $t, \mathrm{~A}$ is at threshold, $\sim \mathrm{A}$ is below, and $\sim \mathrm{B}$ hit threshold before $t$ ahead of $\mathrm{B}$, with likelihood

$$
L_{3}(\mathrm{YES}, t)=f_{\mathrm{A}}(t) \cdot S_{\sim \mathrm{A}}(t) \cdot q(\sim \mathrm{B}, \mathrm{B}, t) \text {. }
$$

4. At time $t$, B is at threshold, $\sim \mathrm{B}$ is below, and $\sim \mathrm{A}$ hit threshold before $t$ ahead of A, with likelihood

$$
L_{4}(\mathrm{YES}, t)=f_{b}(t) \cdot S_{\sim \mathrm{B}}(t) \cdot q(\sim \mathrm{A}, \mathrm{A}, t) .
$$

The overall likelihood of a YES response is then

$$
L(\mathrm{YES}, t)=L_{1}(\mathrm{YES}, t)+L_{2}(\mathrm{YES}, t)+L_{3}(\mathrm{YES}, t)+L_{4}(\mathrm{YES}, t) .
$$

\section{Likelihood of a NO Response}

For a NO response, the two cases are as follows:

1. At time $t, \sim \mathrm{A}$ is at threshold, $\mathrm{A}$ is below threshold, and $\sim \mathrm{B}$ hit threshold before $t$ ahead of $\mathrm{B}$, with likelihood

$$
L_{1}(\mathrm{NO}, t)=f_{\sim \mathrm{A}}(t) \cdot S_{\mathrm{A}}(t) \cdot q(\sim \mathrm{B}, \mathrm{B}, t) .
$$

2. At time $t, \sim \mathrm{B}$ is at threshold, $\mathrm{B}$ is below threshold, and $\sim \mathrm{A}$ hit threshold before $t$ ahead of $\mathrm{A}$, with likelihood

$$
L_{2}(\mathrm{NO}, t)=f_{\sim \mathrm{B}}(t) \cdot S_{\mathrm{B}}(t) \cdot q(\sim \mathrm{A}, \mathrm{A}, t) .
$$

The overall likelihood of a NO response is then

$$
L(\mathrm{NO}, t)=L_{1}(\mathrm{NO}, t)+L_{2}(\mathrm{NO}, t) .
$$

